\title{
Reference values of inspiratory spirometry for Finnish adults
}

\author{
Kainu, Annette
}

2018

Kainu , A , Timonen , K L , Vanninen , E \& Sovijarvi , A R 2018 , ' Reference values of inspiratory spirometry for Finnish adults ', Scandinavian Journal of Clinical \& Laboratory Investigation , vol. 78 , no. 4 , pp. 245-252 . https://doi.org/10.1080/00365513.2018.1439185

http://hdl.handle.net/10138/303903

https://doi.org/10.1080/00365513.2018.1439185

unspecified

publishedVersion

Downloaded from Helda, University of Helsinki institutional repository.

This is an electronic reprint of the original article.

This reprint may differ from the original in pagination and typographic detail.

Please cite the original version. 


\title{
Scandinavian Journal of Clinical and Laboratory
} Investigation

\section{Reference values of inspiratory spirometry for Finnish adults}

\author{
Annette Kainu, Kirsi L. Timonen, Esko Vanninen \& Anssi R. Sovijärvi
}

To cite this article: Annette Kainu, Kirsi L. Timonen, Esko Vanninen \& Anssi R. Sovijärvi (2018) Reference values of inspiratory spirometry for Finnish adults, Scandinavian Journal of Clinical and Laboratory Investigation, 78:4, 245-252, DOI: 10.1080/00365513.2018.1439185

To link to this article: https://doi.org/10.1080/00365513.2018.1439185

曲 Published online: 07 Mar 2018.

Submit your article to this journal

Џ Article views: 29

View Crossmark data \lceil 


\title{
Reference values of inspiratory spirometry for Finnish adults
}

\author{
Annette Kainu ${ }^{\mathrm{a}}$, Kirsi L. Timonen ${ }^{\mathrm{b}}$, Esko Vanninen ${ }^{c}$ and Anssi R. Sovijärvi ${ }^{\mathrm{d}}$ \\ ${ }^{a}$ HUCH Heart and Lung Center, Helsinki University Hospital and University of Helsinki, Helsinki, Finland; ${ }^{b}$ Department of Clinical Physiology \\ and Nuclear Medicine, Central Finland Health Care District, Jyväskylä, Finland; 'Science Center, Kuopio University Hospital, Kuopio, Finland; \\ ${ }^{\mathrm{d} D e p a r t m e n t}$ for Clinical Physiology and Functional Imaging, Helsinki University Hospital and University of Helsinki, Helsinki, Finland
}

\begin{abstract}
Inspiratory spirometry is used in evaluation of upper airway disorders e.g. fixed or variable obstruction. There are, however, very few published data on normal values for inspiratory spirometry. The main aim of this study was to produce reference values for inspiratory spirometry for healthy Finnish adults. Inspiratory spirometry was preplanned to a sample of the Finnish spirometry reference values sample. Data was successfully retrieved from 368 healthy nonsmoking adults (132 males) between 19 and 83 years of age. Reference equations were produced for forced inspiratory vital capacity (FIVC), forced inspiratory volume in one second (FIV1), FIV1/FIVC, peak inspiratory flow (PIF) and the ratios of FIV1/ forced expiratory volume in one second and PIF/peak expiratory flow. The present values were compared to PIF values from previously used Finnish study of Viljanen et al. (1982) reference values and Norwegian values for FIV1, FIVC and FIV1/FIVC presented by Gulsvik et al. (2001). The predicted values from the Gulsvik et al. (2001), provided a good fit for FIVC, but smaller values for FIV1 with mean 108.3 and $109.1 \%$ of predicted values for males and females, respectively. PIF values were 87.4 and $91.2 \%$ of Viljanen et al. (1982) predicted values in males and females, respectively. Differences in measurement methods and selection of results may contribute to the observed differences. Inspiratory spirometry is technically more demanding and needs repeatability criteria to improve validity. New reference values are suggested to clinical use in Finland when assessing inspiratory spirometry. Utility of inspiratory to expiratory values indices in assessment of airway collapse need further study.
\end{abstract}

\section{ARTICLE HISTORY}

Received 18 June 2017

Revised 4 January 2018

Accepted 7 February 2018

\section{KEYWORDS}

Spirometry; reference values; respiratory function tests; lung; inhalation

\section{Introduction}

Expiratory flow-volume spirometry is one of the most widely used lung function measurements. It is mostly indicated to measure ventilatory capacity in forced expiration, forced vital capacity (FVC) and airflow limitation in peripheral intrathoracic airways. Inspiratory flow-volume spirometry, which is not carried out as a screening for lung function test, can give additional information for assessment of central airway obstruction, fixed or variable, e.g. at larynx [1-3]. Obstruction in the upper and central airways can cause dyspnea and sometimes inspiratory stridor also [1,2]. In 1969 Miller and Hyatt [1,3] presented a categorization of three characteristic patterns of flow-volume loops for major airways obstruction: fixed lesions with equally reduced peak inspiratory and expiratory flow, variable extrathoracic lesions with predominantly reduced inspiratory flow and variable intrathoracic lesions with predominantly reduced expiratory flow . Extrathoracic lesions were at the larynx or above the level of the suprasternal notch and intrathoracic lesions were at or below the level of the suprasternal notch [3].

Fixed extrathoracic obstruction may be induced by a variety of conditions like bilateral vocal fold paresis and laryngeal tumours $[1,2,4]$. Variable extrathoracic obstruction can be caused by, e.g. unilateral vocal cord paresis, polyps in the larynx or vocal cords and vocal cord dysfunction (VCD) $[1,3,5-9]$. VCD is very difficult to diagnose and often requires provocation during laryngoscopy to confirm diagnosis [8]. At the symptomatic stage, it can produce a characteristic inspiratory pattern of decreasing flow during inhalation and truncated inspiratory curve, which reduces forced inspiratory flow at 25\% of inspired volume (MIF25) in relation to forced inspiratory flow at $75 \%$ of inspired volume (MIF75) [8-10]. A normal inspiratory spirogram does not exclude VCD. Since causes of inspiratory flow limitation may be variable and difficult to reproduce, if more than one abnormal inspiratory curve is recorded, structural and functional evaluation has been recommended to exclude intraand extrathoracic upper airway obstruction $[8,9]$.

Studies on inspiratory spirometry date mostly to early $1970 \mathrm{~s}$ and is thus influenced by technical development of measuring equipment. Earlier studies used separate peak expiratory flow (PEF) measurements using a Wright peak flow meter adapted also to measure peak inspiratory flow (PIF) [2,6]. Since flow-values were mostly measured from the tracings, values such as mid-vital capacity (mid-VC) flow ratio, measured from the flow-volume tracing at the volume corresponding to $50 \%$ of slow vital capacity, were used $[2-4,11]$. These values are not readily available from 
modern computerized spirometry systems, in which the ratio of PIF/PEF or forced inspiratory volume in one second (FIV1)/forced expiratory volume in one second (FEV1) are readily available and could have diagnostic value in different types of central airway obstructions $[2,8,12]$. The European Respiratory Society (ERS)/American Thoracic Society (ATS) Task Force in 2005 referred to the use of maximum inspiratory flow at $50 \%$ of FVC (MIF50) to maximum expiratory flow at $50 \%$ of FVC (MEF50) with values below one signaling variable extrathoracic obstruction and values above one intrathoracic obstruction [13]. In some other studies, however, the use of MEF50/MIF50 has also been evaluated, but found to have high variability, low repeatability and poor diagnostic predictive value $[9,11,14]$. However, reference values of inspiratory spirometry are lacking for Caucasian people hence determining what constitutes normal is essential.

The recently published multi-ethnic reference values of spirometry (GLI2012) [15] do not include inspiratory measurements. A Scandinavian study by Gulsvik et al. [16] represents only inspiratory reference values available, which included only FIV1 and forced inspiratory vital capacity (FIVC), but did not include any PIF measurements. In Finland, previously used Viljanen et al. [17] reference values from 1982 for spirometry included PIF and a ratio of PIF to MEF50 . The clinical utility of the latter has not been established, but in clinical practice the inspiratory spirometry curve is usually visually inspected and a relationship of PIF to PEF can be assessed. No published data is available, on the interpretation of the PIF/PEF ratio and normal values.

The main aim of this study was to produce reference values of healthy non-smoking Finnish adults for the main variables measured with inspiratory spirometry: FIVC, FIV1, FIV1/FIVC, PIF and the ratios of FIV1/FEV1 and $\mathrm{PIF} / \mathrm{PEF}$.

\section{Materials and methods}

The protocol for spirometry reference values project has been published before [18]. All study participants were native Finns. Uniform inclusion and exclusion criteria were applied to include healthy and non-smoking subjects. Only lifelong never smokers without occupational exposure to vapors, gases, dusts or fumes and with normal weight (body mass index between 20 and $25 \mathrm{~kg} / \mathrm{m}^{2}$ ) were included in this study. Approvals of the study protocols were obtained from Helsinki University Central Hospital Coordinating Ethics Committee and Research Ethics Committee for the Hospital District of Northern Savo (for Kuopio and Tampere) and Länsi-Pohja Central Hospital Ethics Committee (for Kemi). A written informed consent was obtained from all participants.

At all centers, namely Helsinki, Kemi, Kuopio and Tampere, inspiratory spirometry measurements were originally planned. In Kuopio, every fourth subject was invited to complete a separate measurement of forced inspiratory spirometry after expiratory spirometry. In Helsinki and Kemi, all subjects participating in the population based studies were included, but since inspiratory spirometry was technically more demanding, only a smaller proportion of the healthy nonsmoking adults managed to complete a technically satisfactory measurement. Inspiratory spirometry was completed in conjunction with the expiratory spirometry in loops. If the participant was not able to perform satisfactory loops, inspiratory spirometry was discarded. From Tampere and Kemi, the data could not be satisfactorily verified for quality control and hence were excluded from this study. Only spirometry measurements that could be quality controlled from original printouts were accepted.

Identical Vmax $22 \mathrm{D}$ spirometers (heated wire flow transducer spirometer) were used in each study center (Sensor Medics Corporation, Yorba Linda, CA). Spirometers were calibrated every day in the morning. All the measurements were done following the 1994 ATS Standard [19]. Spirometry standards do not have repeatability or quality control criteria for inspiratory spirometry $[13,19,20]$. All measurements were visually controlled and to be acceptable the best inspiratory curve was required to be with one clear peak with good effort. No preconceived PIF or FIV1 limits were imposed. From acceptable curves, the highest FIVC, FIV1 and PIF were selected. If inspiratory time was under $1 \mathrm{~s}$, the spirometer did not report a FIV1. In these situations, FIV1 was considered equal to FIVC. The ratio of FIV1/ FIVC was calculated from the selected best values. Similar repeatability criteria were considered for inspiratory maneuvers as a set for expiratory measurements with two best FIVC and FIV1 values aimed within $200 \mathrm{ml}$ and two best PIF within $20 \%$. Three acceptable measurements were aimed for with a maximum of eight efforts. Repeatability was required at measurements undertaken in Kuopio and to be accepted for the study there had to be at least two parallel inspiratory measurements fulfilling the repeatability criteria. For Helsinki, the curves were individually manually checked since the software automatically selected the inspiratory values of best expiratory maneuver as the best inspiratory maneuver. Repeatability criteria for inspiratory spirometry could not be implemented at the time of the actual measurements, but the same inspiratory value repeatability criteria as in Kuopio were implemented when screening acceptable spirometry's for this study.

The variables analyzed were FIVC, FIV1, FIV1/FIVC, PIF, PIF/PEF and FIV1/FEV1. Various modelling approaches were evaluated including the Generalized Additive Model for Location, Scale and Shape (GAMLSS) model used in the development of the Global Lung Function Initiative (GLI) reference values. The simplest model resulting in highest degree of determination was chosen. Predictors were included in the model in a stepwise manner and retained in the final model if they improved the degree of determination significantly. Predicted mean and 5th percentile lower limit of normal (LLN) were calculated using a linear regression model using the Equation (1):

$$
\mathrm{f}=\mathrm{a} 0+\mathrm{a} 1 \times \operatorname{sex}+\mathrm{a} 2 \times \text { age }+\mathrm{a} 3 \times \ln (\mathrm{age})+\mathrm{a} 4 \times \text { height }
$$

with a0, a1, a2, a3 and a4 are the regression coefficients. Measured values were presented as percent of predicted values from this study and the currently used Viljanen et al. [17] 
reference value for PIF and published reference values of Gulsvik et al. [16] for FIVC, FIV1, and FIV1/FIVC were compared using the Wilcoxon Signed Ranks test.

All statistical analyses were conducted using the statistical program SPSS (IBM SPSS Statistics for Macintosh, Version 22.0. Armonk, NY: IBM Corp.). Chi-squared test was used for categorical comparisons. Normality was tested with Shapiro-Wilks test for normality and comparison of continuous variables was done with Students t-test for normally distributed variables and Wilcoxon Signed Rank test for non-normally distributed variables. A $p$ value of .05 was chosen for all comparisons. Data on differences between predicted values of PIF from this study and the currently used Viljanen et al. [17] reference values are presented as Bland-Altman plot [21].

\section{Results}

Descriptive statistics of the study population are presented in Table 1 . Since Kuopio specifically recruited healthy older subjects in the reference values study, the participating subjects in Kuopio were older 52.7 (SD 18.0) vs. 47.5 (SD 12.8) years $(p=.001)$ and shorter 167.3 (SD 9.4) vs. 169.6 (SD $9.0) \mathrm{cm}(p=0.016)$ than in Helsinki. Between-center differences were evaluated by modelling PIF for the combined data and for both centers separately as illustrated in Figure 1 for males and females. The crude PIF values for Helsinki were larger and in relative to Viljanen et al. reference values [17] smaller than in Kuopio, but after taking into account the sex, age and height of the subjects, these differences were not significant.

The measured values of FIVC, FIV1, PIF and the ratios of FIV1/FVC, FIV1/FEV1 and PIF/PEF are shown in Figure 2 stratified by sex. Regression coefficients for the prediction models for FIVC, FIV1, PIF, FIV1/FVC, FIV1/FEV1 and PIF/PEF are presented in Table 2.

There are very little published data on inspiratory spirometry and in comparison to previously published and used values can thus be presented only for two studies: Gulsvik et al. (2001) for FIV1, FIVC and FIV/FIVC and Viljanen et al. (1982) for PIF [16,17]. In Figure 3 the predicted values from Gulsvik et al. [16] are compared to models presented in this study for average height of males and females for FIV1, FIVC and FIV1/FIVC. The predicted values from this study are slightly larger in females and in older adults. The
PIF values predicted by Viljanen et al. [17] are slightly larger especially in younger adults, but the difference diminishes with age as illustrated in Figure 4(a). In the study participants, the Viljanen et al. [17] predicted values produced consistently larger values than the predicted values from the present study, with a larger difference in subjects with higher PIF values as shown in the Bland-Altman graph in Figure 4(b). The predicted values for the reference values sample from Gulsvik et al. [16] and Viljanen et al. [17] and also the models from the present study are presented in Table 3. The predicted values by Gulsvik et al. [16], provide a good fit for FIVC, but significantly smaller values for FIV1 with a mean 108.3 and $109.1 \%$ of predicted values for males and females, respectively $(p<.001)$. This difference could be partially influenced by measurement methods, which could thereby influence the also found differences in FIV1/FIVC.

\section{Discussion}

Inspiratory spirometry is seldom used, but when used needs reference values to identify deviations from normal values. To date there has been very limited data to define normality. In this study, reference equations for main inspiratory spirometry variables and their ratios to the expiratory equivalents are presented for clinical use.

Inspiratory spirometry is technically more demanding than the expiratory maneuver. Gulsvik et al. [16] have shown in their study that the intraindividual variation in FIV1 is greater than of that in FEV1 and repeatability is difficult to achieve. These results support the need for this finding. Spirometry standards have no repeatability or quality criteria for the inspiratory maneuvers. No preconceived limits for acceptable PIF levels were chosen, but a good curve with one clear peak was required. The resulting PIF levels were lower than expected when compared to previously used Viljanen et al. [17] predicted values. However, the FIV1 and FIVC levels were very close to the values predicted by Gulsvik et al. [16] thus this seems to be a naturally occurring phenomenon and does not necessarily represent poor effort or quality.

In Kuopio, inspiratory measurements were done separately as loops, meaning that the subject did a forced inspiratory maneuver immediately after the forced expiratory maneuver, with repeatability criteria similar to the expiratory

Table 1. Descriptive statistics of the study population. Data presented as mean (SD).

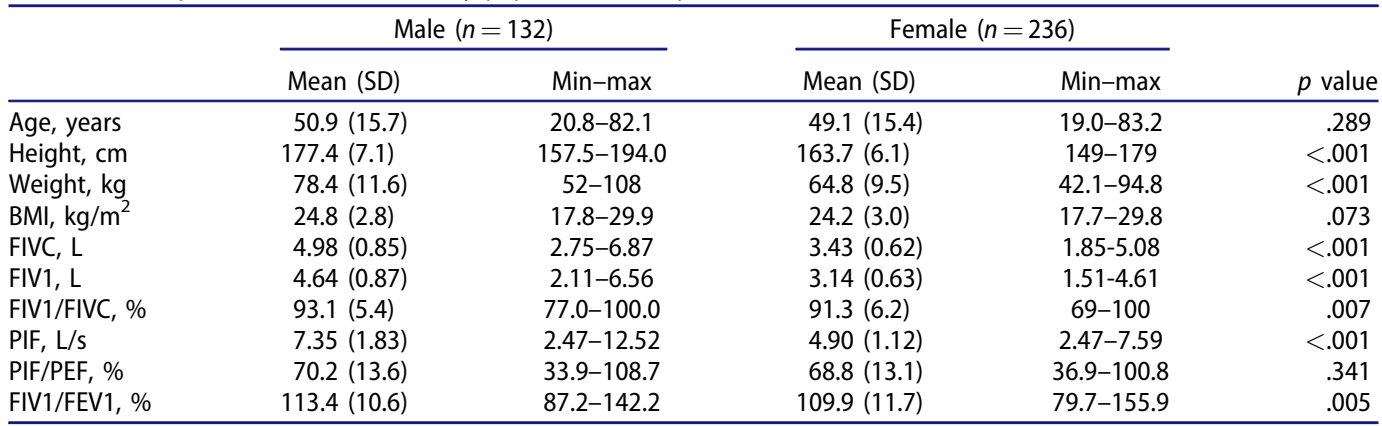

BMI: body mass index; FIVC: forced inspiratory vital capacity; FIV1: forced inspiratory volume in one second; PIF: peak inspiratory flow; PEF: peak expiratory flow; FEV1: forced expiratory volume in one second. 

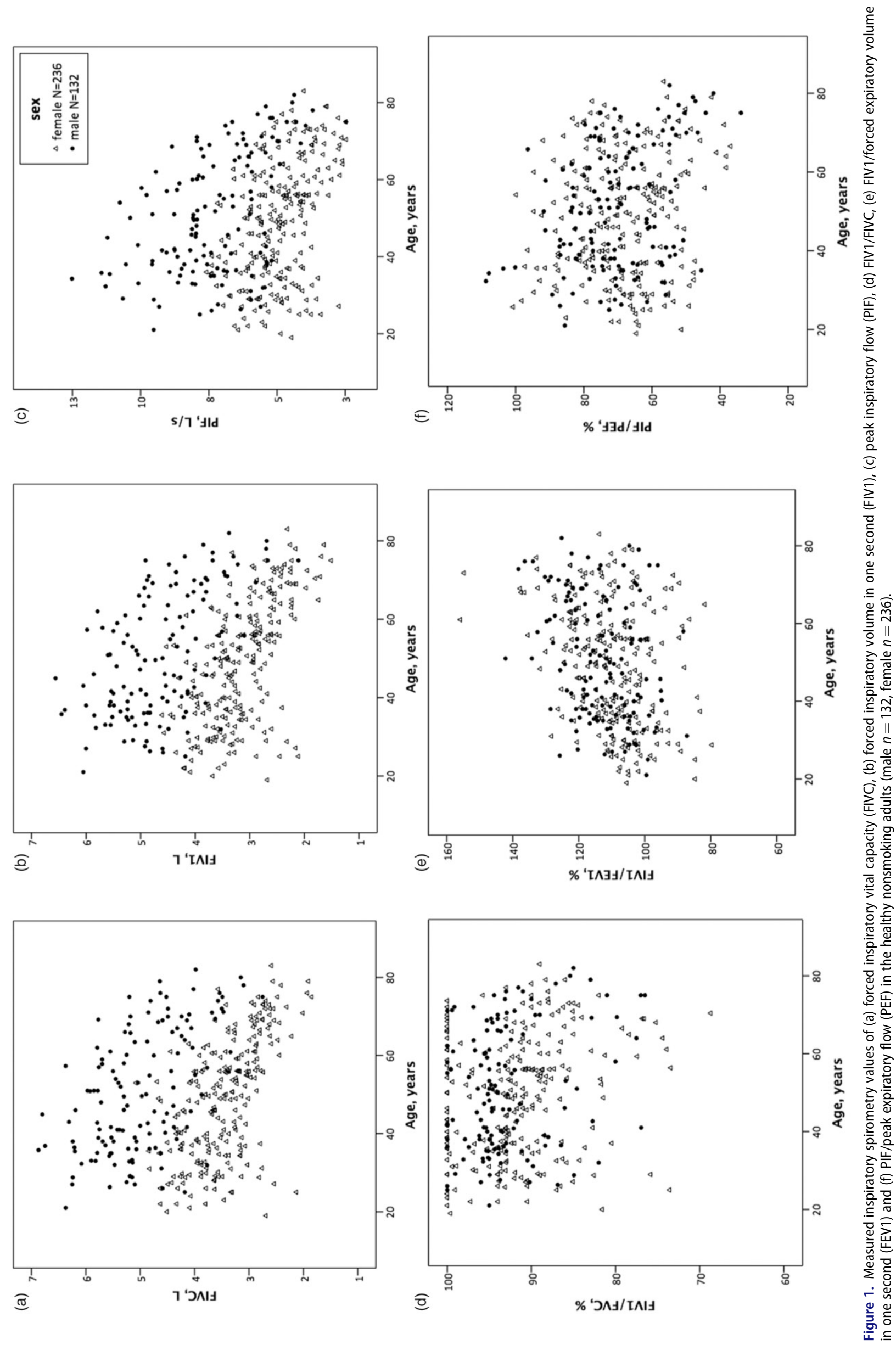

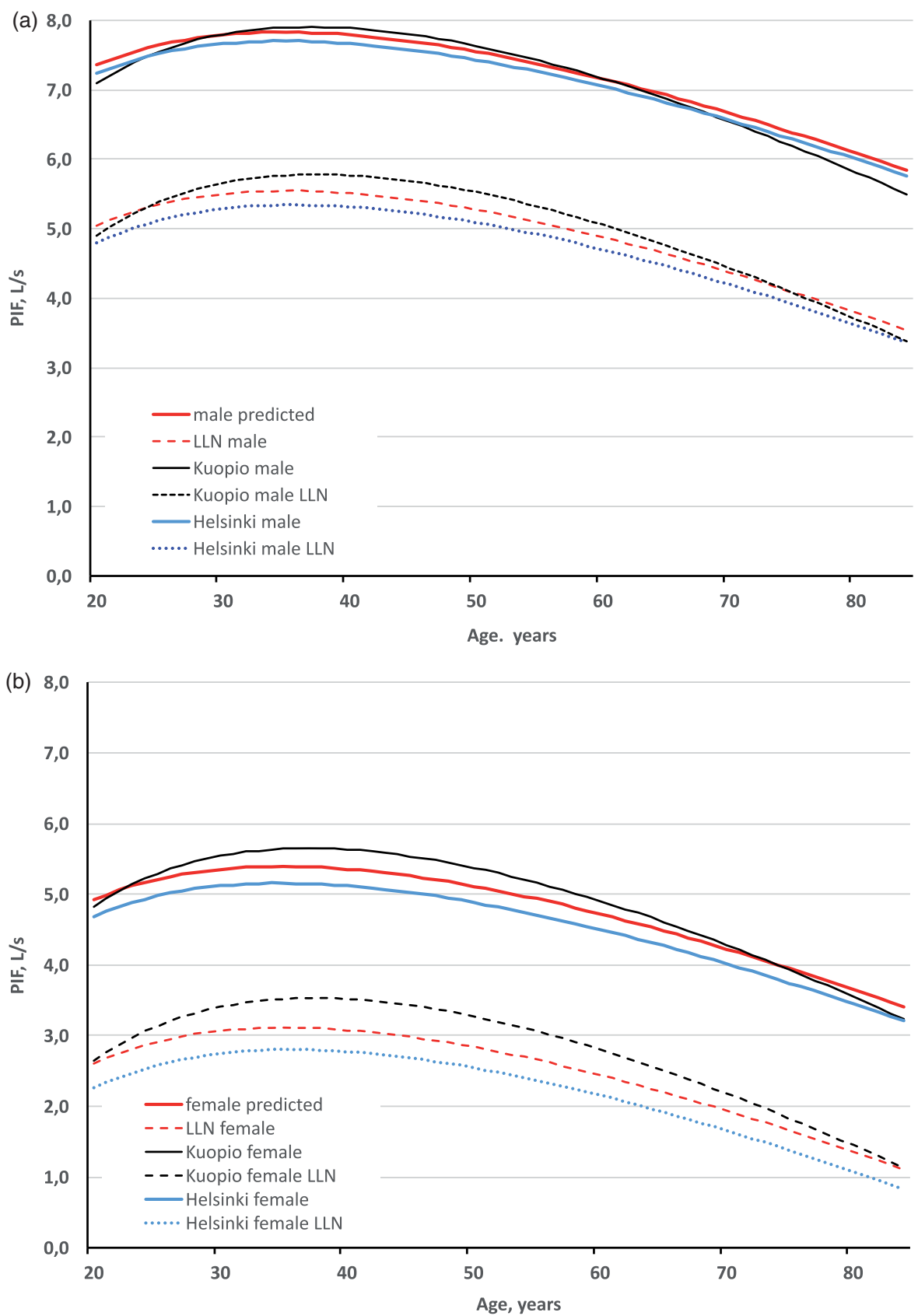

Figure 2. Predicted peak inspiratory flow (PIF) and lower limit of normal (LLN) for the combined sample and for Kuopio and Helsinki centers modelled separately for (a) males $(177 \mathrm{~cm})$ and $(\mathrm{b})$ females $(164 \mathrm{~cm})$ in height.

maneuvers applied to inspiratory values. In Helsinki, the inspiratory maneuvers were done as loops in conjunction with the expiratory spirometry with best curves selected based on expiratory maneuver quality and repeatability criteria. In the Norwegian study by Gulsvik et al. [16] inspiratory measurements were also done as loops and selection of best maneuvers was done primarily based on the expiratory limb of the measurement. The spirometry software does not permit selecting best inspiratory and expiratory parts from separate maneuvers. In this study, the best inspiratory maneuvers in Helsinki were thus selected manually afterwards by visual inspection of all curves. Equivalent repeatability criteria were strived for, but could not be effectively enforced. The predicted values from Kuopio measurements were slightly higher, but the difference between the centers was not significant after controlling for sex, age and height of the subjects. However, since the inspiratory measurements as loops were significantly more difficult for the subjects, it would seem reasonable to recommend doing the inspiratory maneuvers, when indicated, as separate measurements after establishing the subjects FEV1 and peak flow to ensure accurate baseline for comparison. Since measurement variability is even larger than in expiratory spirometry, repeatability criteria for the measurements should be also defined to improve the validity of measurements done at different locations or time points.

The predicted values from the Norwegian study by Gulsvik et al. [16], provided a good fit for FIVC in this sample, but smaller values for FIV1 with a mean of 108.3 and $109.1 \%$ of predicted values for males and females, 
respectively. Differences in measurement methods and selection of results may contribute to this observed difference. In the study by Gulsvik et al. [16] the quality criteria had been selected to so that the FIVC needs to be within $200 \mathrm{ml}$ of the largest FVC. Smaller proportion of the subjects completed acceptable FIV1 than FIVC, but there is no indication in study methods how maneuvers with inspiratory time under one second were handled. In this study, it was decided from the outset that FIVC would be substituted for FIV1, if the inspiratory time in a good maneuver was under one second. This may have influenced the observed differences in FIV1 and FIV1/FIVC between these studies.

In this study several indices reflecting differences between inspiratory and expiratory spirometry are presented. The ratio PIF/PEF is used in clinical practice and reflects possible

Table 2. Regression coefficients for reference equations for mean and lower limit of normal (LLN) for the inspiratory spirometry variables.

\begin{tabular}{lcccccc}
\hline Variable & Constant & Sex $^{\mathrm{a}}$ & Age & Ln (age) & Height & $R_{\text {adj }}^{2}$ \\
\hline FIVC, L & & & & & & \\
$\quad$ Mean & -7.203 & 0.825 & -0.036 & 0.938 & 0.054 & $76.8 \%$ \\
$\quad$ LLN & -8.409 & 0.815 & -0.044 & 1.351 & 0.053 & \\
FIV1, L & & & & & & \\
$\quad$ Mean & -7.711 & 0.847 & -0.045 & 1.307 & 0.049 & $75.0 \%$ \\
$\quad$ LLN & -8.956 & 0.836 & -0.054 & 1.734 & 0.049 & \\
FIV1/FIVC, \% & & & & & & \\
$\quad$ Mean & 61.412 & 1.874 & -0.345 & 12.202 & - & $7.6 \%$ \\
$\quad$ LLN & 46.457 & 1.687 & -0.448 & 17.132 & - & \\
PIF, L/s & & & & & & \\
$\quad$ Mean & -15.892 & 1.500 & -0.107 & 3.719 & 0.072 & $60.1 \%$ \\
$\quad$ LLN & -18.561 & 1.498 & -0.110 & 3.858 & 0.072 & \\
$\begin{array}{l}\text { PIF/PEF, } \% \\
\text { Mean }\end{array}$ & -72.393 & -3.461 & -0.783 & 31.366 & 0.362 & $9.3 \%$ \\
$\quad$ LLN & -103.270 & -3.722 & -1.004 & 41.949 & 0.352 & \\
FIV1/FEV1,\% & & & & & & \\
$\quad$ Mean & 48.529 & 3.055 & -0.148 & 17.880 & - & $12.0 \%$ \\
$\quad$ LLN & 20.525 & 2.705 & -0.341 & 27.112 & - & \\
\hline
\end{tabular}

${ }^{\mathrm{a}}$ Male $=1 ;$ Female $=0$

$R_{\mathrm{adj}}^{2}$ : degree of determination; LLN: lower limit of normal; FIVC: forced inspiratory vital capacity; FIV1: forced inspiratory volume in one second; PIF: peak inspiratory flow.
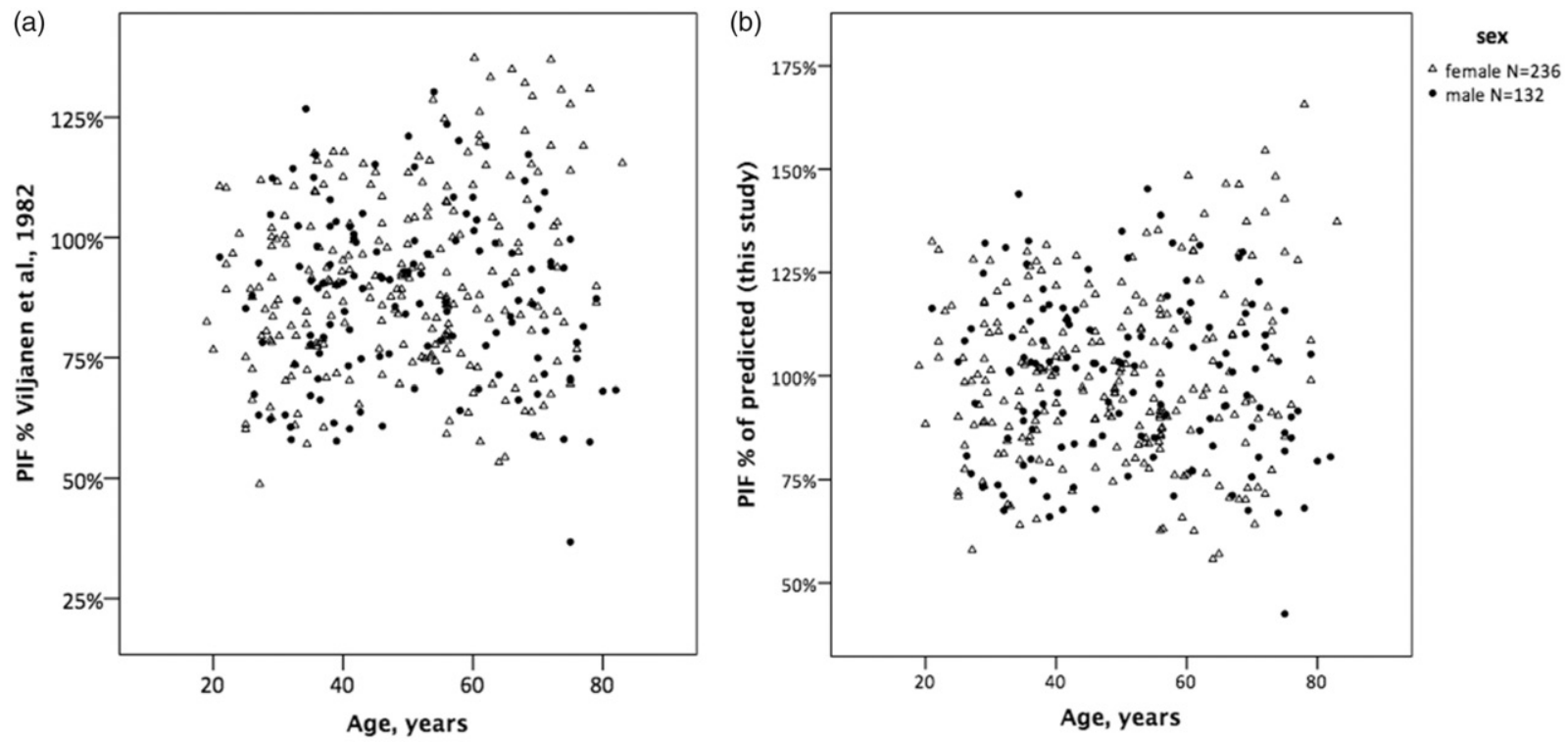

Figure 3. Measured peak inspiratory flow (PIF) values expressed as percent predicted of (a) Viljanen et al., 1982 reference values, (b) predicted values derived from this study for healthy nonsmoking adults. extrathoracic airway stenosis, although there are very few published studies using spirometry [5]. The relationships between FIV1/FEV1 or FIVC/FVC are even less studied, but it is hypothesized that these indices could prove useful in selected clinical situations e.g. in the evaluation of variable extra- or intrathoracic airway obstruction or collapse in phenotyping severe asthma $[2,4,8,12]$. Further study is needed to assess their usability. Since the reproducibility of the inspiratory values has been found poorer than expiratory spirometry even with FIV1 and FIVC, the measurement of MIF50 and MEF50, both of which are highly dependent on volume changes in the respective inspiratory and expiratory maneuvers, the MIF50/MEF50 ratio was not separately modelled [16]. It should also be noted that all the inspiratoryexpiratory ratios also have low degrees of determination in this study.

Since there are very little published data on inspiratory spirometry, these values cannot be compared to other published indices. In the study by Gulsvik et al. [16] FEV1/FIV1 ratio was found to be higher in females than in males while the FIV1/FIVC was lower in females and this was suggested to be caused by men having more respiratory musculature than females. The findings from this study are concurrent with this with males having higher predicted values in FIV1/ FIVC and FIV1/FEV1. Height didn't significantly contribute to the prediction models in these variables and was thus excluded (Table 2). However, in PIF/PEF, height affects predicted values with taller subjects having higher predicted PIF/PEF ratio and the predicted PIF/PEF values were consistently smaller for same height males compared to females.

Clinical studies have evaluated the usefulness of different indices to detect upper airway disease noninvasively from flow-volume spirometry. Modrykamien et al. [14] evaluated four quantitative and three visual criteria, but the only inspiratory flow value used was MIF50, which has high measurement variability. Quantitative criteria showed low sensitivity for detection, but exceeded that of visual criteria 
(a)

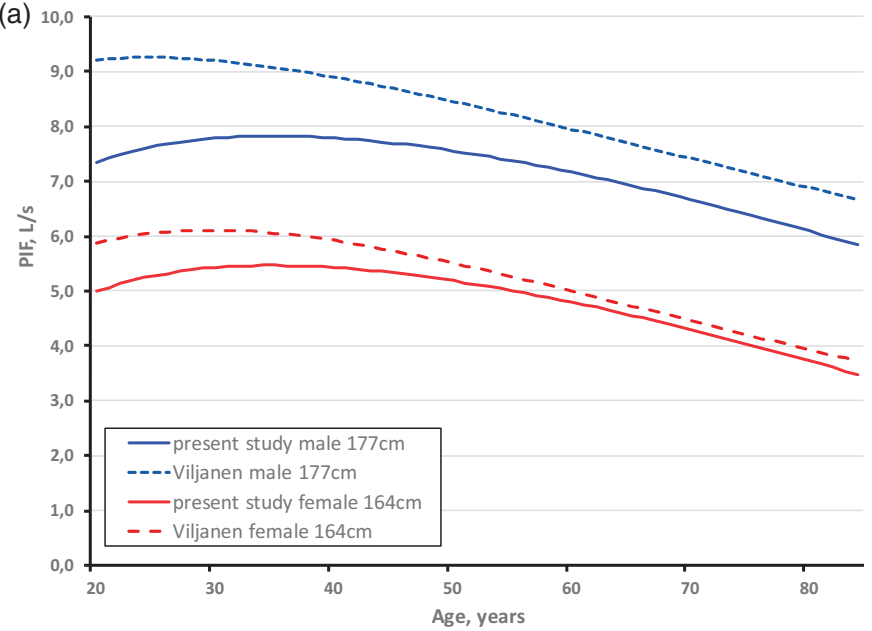

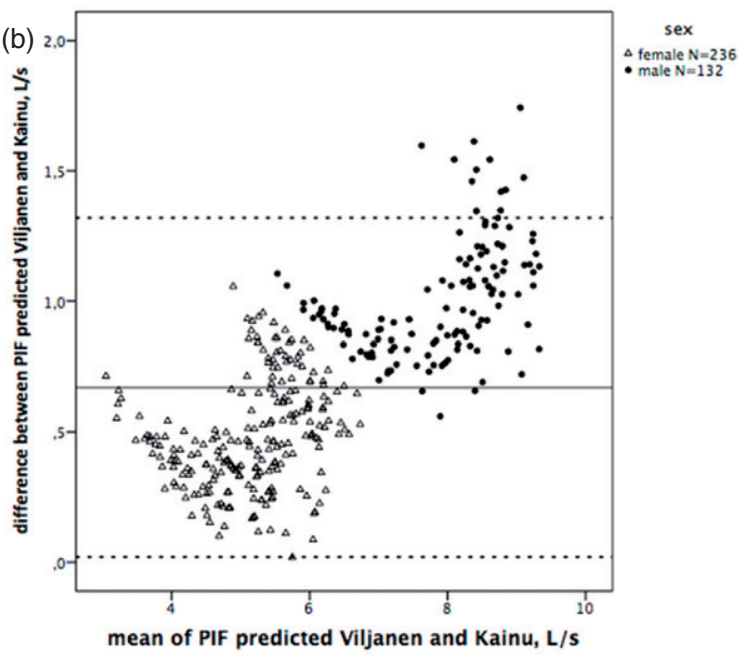

Figure 4. (a) Predicted peak inspiratory flow (PIF) values from Viljanen et al. [17] and values from the present study for males (177 cm) and females (164 cm). (b) Bland-Altman graph [21] showing the difference between PIF predicted with Viljanen et al. [17] reference values and values derived from the present study in relation to their mean. Horizontal solid line indicating the mean and dashed lines indicating the 95th percentile confidence limits.

Table 3. Predicted values in the study population of healthy nonsmoking adults. Comparison between reference models presented with Wilcoxon Signed Rank test.

\begin{tabular}{|c|c|c|c|c|c|}
\hline & \multicolumn{2}{|c|}{ Male $(n=132)$} & \multicolumn{2}{|c|}{ Female $(n=236)$} & \multirow[b]{2}{*}{$p$ value $^{a}$} \\
\hline & Mean (SD) & $95 \% \mathrm{Cl}$ for mean & Mean (SD) & $95 \% \mathrm{Cl}$ for mean & \\
\hline \multicolumn{6}{|l|}{ FIVC } \\
\hline Present study & $99.3 \%(12.6 \%)$ & $97.1-101.5$ & $99.1 \%(11.8 \%)$ & $97.6-100.6$ & Male: .064 Female: .044 \\
\hline \multicolumn{6}{|l|}{ FIV1 } \\
\hline Present study & $100.5 \%(13.9 \%)$ & $98.1-102.9$ & $100.9 \%(14.1 \%)$ & $99.1-102.7$ & \multirow[t]{2}{*}{ Male: $<.001$ Female: $<.001 .001$} \\
\hline Gulsvik et al. [16] & $108.3 \%(17.6 \%)$ & $105.3-111.3$ & $109.1 \%(17.6 \%)$ & $106.8-111.3$ & \\
\hline Gulsvik et al. [16] & $105.9 \%(6.5 \%)$ & $104.7-107.0 \%$ & $109.4 \%(7.6 \%)$ & $108.4-110.4 \%$ & Male: $<.001$ Female: $<.001$ \\
\hline \multicolumn{6}{|l|}{ PIF } \\
\hline Present study & $99.3 \%(19.5 \%)$ & $96.8-102.7 \%$ & $100.3 \%(20.5 \%)$ & $97.6-102.9 \%$ & \multirow[t]{2}{*}{ Male: $<.001$ Female: $<.001$} \\
\hline Viljanen et al. [17] & $87.4 \%(17.6 \%)$ & $84.4-90.5$ & $91.2 \%(18.4 \%)$ & $88.8-93.5$ & \\
\hline \multicolumn{6}{|l|}{$\mathrm{PIF} / \mathrm{PEF}$} \\
\hline Present study & $99.9 \%(17.6 \%)$ & $96.8-102.7$ & $100.1 \%(18.6 \%)$ & $97.7-102.4$ & \\
\hline \multicolumn{6}{|l|}{ FIV1/FEV1 } \\
\hline
\end{tabular}

Cl: confidence interval; FIVC: forced inspiratory vital capacity; FIV1: forced inspiratory volume in one second; FEV1: forced expiratory volume in one second; PIF: peak inspiratory flow; PEF: peak expiratory flow.

${ }^{a}$ Wilcoxon Signed Rank test.

[14]. Suspected VCD is commonly sought to verify with inspiratory spirometry, but poses great challenges as the condition itself is highly variable and produces typical changes to inspiratory flow values only when symptomatic [8]. MIF25/MIF75 ratio was the only index predictive of VCD and even that lost significance after controlling for reproducibility of the inspiratory manouvers [8]. This might be influenced by the high variability of the physiologic phenomenon as no provocation has been used before spirometry to precipitate the findings $[8,9]$. Normal inspiratory spirometry does not exclude VCD and further study is always recommended when more than one abnormal inspiratory maneuver is recorded $[8,9]$. Unilateral fixed vocal cord paralysis has been shown to produce fixed extrathoracic airway obstruction i.e. constantly reduced PIF in relation to PEF [5].

\section{Study limitations}

This study includes 368 adults, of whom only 132 were male. The small study sample poses limitations to applicable modelling approaches and generalizability of the results. However, there are limited data available on inspiratory spirometry and thus this small sample is seen of value to gain some insight to normative values in the inspiratory maneuvers. There are also very limited studies on the quality criteria, repeatability and clinical interpretation of inspiratory spirometry, which would warrant further study beyond the scope of this manuscript.

\section{Conclusion}

Inspiratory spirometry is technically more demanding than expiratory spirometry and needs quality criteria to add 
validity. Prediction equations are given for main inspiratory spirometry variables and also for their ratios to the respective expiratory values. Usability of these indexes requires further study.

\section{Acknowledgements}

The HEVI Project centers in Helsinki and Kuopio are acknowledged for conducting the original reference values study producing spirometry measurements of the study subjects.

\section{Disclosure statement}

No potential conflict of interest was reported by the authors.

\section{Funding}

The HEVI Project has been funded from Tampere Tuberculosis Foundation. The FinEsS-Helsinki study has received funding from the Special Governmental Subsidy for Health Sciences Research (project codes TYH1235, TYH 2303, TYH 4251, and TYH 2013354). Dr Annette Kainu has received a research grant from the Finnish AntiTuberculosis Foundation, Jalmari and Rauha Ahokas Foundation and funding from the Special Governmental Subsidy for Health Sciences Research (HUCH Heart and Lung Center).

\section{References}

[1] Miller RD, Hyatt RE. Obstructing lesions of the larynx and trachea: clinical and physiological characteristics. Mayo Clin Proc. 1969;44:145-161.

[2] Clark TJH. Inspiratory obstruction. Br Med J. 1970; 3:682-684.

[3] Miller RD, Hyatt RE. Evaluation of obstructing lesions of the trachea and larynx by flow-volume loops. Am Rev Respir Dis. 1973;108:475-481.

[4] Engström H, Grimby G, Söderholm B. Dynamic spirometry in patients with tracheal stenosis. Acta Med Scand. 1964;176: 329-334.

[5] Saarinen A, Rihkanen H, Malmberg LP, et al. Disturbances in airflow dynamics and tracheal sounds during forced and quiet breathing in subjects with unilateral vocal fold paralysis. Clin Physiol. 2001; 6:712-717.
[6] Empey DW. Assessment of upper airways obstruction. Br Med J. 1972;3:503-505.

[7] Herzog H, Keller R, Allgöwer M. Special methods of diagnosing and treating obstructive diseases of the central airways. Chest. 1971;60:49-67.

[8] Watson MA, King CS, Holley AB, et al. Clinical and lung-function variables associated with vocal cord dysfunction. Respir Care. 2009;54:467-473.

[9] Sterner JB, Morris MJ, Sill JM, et al. Inspiratory flow-volume curve evaluation for detecting upper airway disease. Respir Care. 2009;54:461-466.

[10] Owens GR, Murphy DMF. Spirometric diagnosis of upper airway obstruction. Arch Intern Med. 1983;143:1331-1334.

[11] Rotman HH, Liss HP, Weg JG. Diagnosis of upper airway obstruction by pulmonary function testing. Chest. 1975;68: 796-799.

[12] García-Pachón E, Casan P, Sanchis J. Indices of upper airway obstruction in patients with simultaneous chronic airflow limitation. Respir. 1994;61:121-125.

[13] Pellegrino R, Viegi G, Brusasco V, et al. ATS/ERS Task Force: standardisation of lung function testing. Interpretative strategies for lung function tests. Eur Respir J. 2005;26:948-968.

[14] Modrykamien AM, Gudavalli R, McCarthy K, et al. Detection of upper airway obstruction with spirometry results and the flow-volume loop: a comparison of quantitative and visual inspection criteria. Respir Care. 2009;54:474-479.

[15] Quanjer PH, Stanojevic S, Cole TJ, et al. Multi-ethnic reference values for spirometry for the 3-95-yr age range: the global lung function 2012 equations. Eur Respir J. 2012;40: 1324-1343.

[16] Gulsvik A, Tosteson T, Bakke P, et al. Expiratory and inspiratory forced vital capacity and one-second forced volume in asymptomatic never-smokers in Norway. Clin Physiol Funct Physiol. 2001;21:648-660.

[17] Viljanen AA, Halttunen PK, Kreus KE, et al. Spirometric studies in non-smoking, healthy adults. Scand J Clin Lab Invest. 1982;42(Suppl. 159):5-20.

[18] Kainu A, Timonen K, Toikka J, et al. Reference values of spirometry for Finnish adults. Clin Physiol Funct Imaging. 2016; 6:346-358

[19] American Thoracic Society. Standardization of spirometry. 1994 Update. Am J Respir Crit Care Med. 1995;152:1107-1136.

[20] Miller MR, Hankinson J, Brusasco V, et al. ATS/ERS Task Force: standardisation of lung function testing. Standardisation of spirometry. Eur Respir J. 2005;26:319-338.

[21] Bland JM, Altman DG. Statistical methods for assessing agreement between two methods. Lancet. 1986; 1:307-310. 